LETTER TO THE EDITOR

\title{
Lower entropy bounds and particle number fluctuations in a Fermi sea
}

\author{
Israel Klich \\ Department of Physics, Caltech, Pasadena, CA 91125, USA \\ E-mail: klich@caltech.edu
}

Received 21 October 2005, in final form 30 November 2005

Published 11 January 2006

Online at stacks.iop.org/JPhysA/39/L85

\begin{abstract}
In this letter we demonstrate, in an elementary manner, that given a partition of the single particle Hilbert space into orthogonal subspaces, a Fermi sea may be factored into pairs of entangled modes, similar to a BCS state. We derive expressions for the entropy and for the particle number fluctuations of a subspace of a Fermi sea, at zero and finite temperatures, and relate these by a lower bound on the entropy. As an application we investigate analytically and numerically these quantities for electrons in the lowest Landau level of a quantum Hall sample.
\end{abstract}

PACS numbers: 03.67.Mn, 05.70.-a, 05.30.Fk

The study of quantum many particle states, when measurements are only applied to a given subsystem, are at the heart of many questions in physics. Examples where the entropy of such subsystems is interesting range from the quantum mechanical origins of black hole entropy, where the existence of an event horizon thermalizes the field density matrix inside the black hole [1, 2] to entanglement structure of spin systems [3]. In this work we address the relation of entanglement entropy of fermions with the fluctuations in the number of fermions.

A general treatment of a BCS-like factorization of a Gaussian state on a bi-partite system was carried out in [4]. Here we concentrate on a particular case and show in an elementary way how a given subspace of the single particle Hilbert space, a Fermi sea, may be factorized into pairs of entangled modes in and out of the subspace, thereby writing the state as a BCS state. We then use this construction to calculate various properties of the fermions in one of the subsystems.

While upper bounds on entropy were the subject of numerous investigations, especially since Bekenstein's bound [5], lower bounds on entropy are less known.

We show that given a 'Fermi sea', the entropy of the ground state, restricted to a particular subspace $A$ of the single particle space, relates to the particle number fluctuations in the 
subspace via the inequality:

$$
S_{A} \geqslant(4 \log 2) \Delta N_{A}^{2} \geqslant-(8 \log 2)\left\langle\left\langle N_{A}^{4}\right\rangle\right\rangle,
$$

where $S_{A}$ is the entropy associated with the subspace $A, \Delta N_{A}^{2}$ are the fluctuations in the particle number in $A$ and $\left\langle\left\langle N_{A}^{4}\right\rangle\right\rangle$ is the fourth cummulant of particle number ${ }^{1}$. The importance of this result lies in the fact that particle fluctuations are, in principle, measurable, and are fundamentally related to the quantum noise in various systems. On the technical side, note that the right-hand side has the advantage of being easy to calculate analytically in a wider class of problems.

We start by examining the ground state of non-interacting fermions, in arbitrary external potential, when measurements are applied to a given part of the space. The basic example is a Fermi sea or a Dirac sea where we are interested in the relative entropy of a given region of space, and in fluctuations in the number of particles there, but one may also consider entanglement in Fermion traps (Fermi degeneracy of potassium atoms $\left({ }^{40} \mathrm{~K}\right.$ ) has been observed by DeMarco and Jin [6]).

The discussion is also relevant for systems which behave like a non-interacting Fermi gas, as in problems of transport at the zero temperature limit $^{2}$, in ideal metals, where transport may be approximated well within a non-interacting theory, due to good screening. In an ideal single channel conductor the analogy is done by mapping excitations that travel at the Fermi velocity to a time-energy coordinate representation in discussion of quantum pumps [7-9]. The analogy is especially manifest in the problem of switching noise [10].

The ground state of a noninteracting Fermi gas, containing $N$ particles, is obtained by occupying the allowed states $\phi_{i} \in H$ ( $H$ is the single particle Hilbert space) up to energy $E_{f}$, i.e.

$$
|g s\rangle=\prod_{E\left(\phi_{i}\right)<E_{f}} \psi^{\dagger}\left(\phi_{i}\right)|0\rangle
$$

where $\psi^{\dagger}$ are creation operators which satisfy the usual canonical anti-commutation relations (CAR):

$\left[\psi\left(\phi_{i}\right), \psi^{\dagger}\left(\phi_{j}\right)\right]_{+}=\left\langle\phi_{i}, \phi_{j}\right\rangle \quad\left[\psi\left(\phi_{i}\right), \psi\left(\phi_{j}\right)\right]_{+}=\left[\psi\left(\phi_{i}\right)^{\dagger}, \psi^{\dagger}\left(\phi_{j}\right)\right]_{+}=0$.

The state $|g s\rangle$ or the 'Fermi sea' is a typical ground state for a large class of Hamiltonians (sometimes it is necessary to carry a suitable Bogolubov transformation). It can also describe spin chains via the Jordan-Wigner transformation.

Let $A$ be a subspace of the single particle Hilbert space $H$, so that $H=A \oplus A^{\perp}$ and let $E=\operatorname{span}\left\{\phi_{i} ; 1 \leqslant i \leqslant N\right\}$ be the subspace of occupied single particle states of $H$ (the Fermi sea).

Let $P_{A}$ be the orthogonal projection on $A$. Consider the matrix

$$
M(A)_{i j}=\left\langle P_{A} \phi_{j}, P_{A} \phi_{i}\right\rangle,
$$

with $i, j=1, \ldots, N .^{3} M_{i j}$ is a Hermitian matrix and is diagonalized by a unitary $U: M=U^{\dagger} \operatorname{diag}\left(d_{i}\right) U$.

The new orthonormal modes are defined by

$$
A_{l}=\frac{\sum_{k} U_{l k}^{\dagger} P_{A} \phi_{k}}{\sqrt{d_{l}}}
$$

1 Note that a fourth cummulant may have either sign.

2 The zero temperature state is completely degenerate. Otherwise, we need the condition (for strong degeneracy)

$T \ll E_{F}$.

3 We restrict ourself to states that obey $\left\|P_{A} \phi_{j}\right\|^{2}>0$, otherwise they are already of the required form. 
where the factor $d_{l}$ is the $l$ th eigenvalue of $M$, and serves to normalize the $A_{l}$ with the inner product on $H$. Similarly we take $B_{l}=\frac{\sum_{k} U_{l k}^{\dagger} P_{A}^{\perp} \phi_{k}}{\sqrt{1-d_{l}}}$, which are orthonormal since $M\left(A^{\perp}\right)=I-M(A)$ is diagonalized by $U$ as well. Obviously $A_{i} \in A$ and $B_{i} \in A^{\perp}$.

Since $U$ is unitary we write $E=\operatorname{span}\left\{U^{\dagger} \phi_{i}\right\}$. Using $U_{i l}^{\dagger} \phi_{l}=\sqrt{d_{i}} A_{i}+\sqrt{1-d_{i}} B_{i}$, the ground state may be written as (up to an overall phase)

$$
|g s\rangle=\prod_{i=1}^{N} \Psi^{\dagger}\left(U_{i j}^{\dagger} \phi_{j}\right)|0\rangle=\prod_{i=1}^{N}\left(\sqrt{d_{i}} \Psi^{\dagger}\left(A_{i}\right)+\sqrt{1-d_{i}} \Psi^{\dagger}\left(B_{i}\right)\right)|0\rangle .
$$

Note that the $0 \leqslant d_{i} \leqslant 1$ due to the structure of $M$. We also note that it is not assumed that the dimension of the subspace $A$ is larger than the number of fermions $N$. In this case $\operatorname{dim} A<N$, the matrix $M$ will have some of its eigenvalues vanishing, signaling that some modes of $B$ are occupied with probability one. In general we always have that $\#\left\{d_{i} \neq 0\right\} \leqslant \min (\operatorname{dim} A, N)$. In the simplest case where $A$ is only a one-dimensional subspace, the matrix $M$ will have at most one eigenvalue which differs from zero, which is the probability amplitude of this single mode to be occupied.

In the particular case that $\left[P_{E}, P_{A}\right]=0$ the exercise becomes trivial, as $P_{E}$ and $P_{A}$ can be diagonalized simultaneously. In addition, whenever there is a symmetry operator that commutes with $P_{A}$ and $P_{E}$, then the resultant eigenmodes are invariant under the symmetry.

Note, incidentally, that the ground state may be written also as a BCS state in the following way. Consider the vector $|A\rangle=\prod \psi^{\dagger}\left(A_{i}\right)|0\rangle$, defined by 'filling' the modes in $A$, and redefine $\psi\left(A_{i}\right)=\psi_{h}^{\dagger}\left(A_{i}\right)$, then

$$
|g s\rangle=\prod_{i}\left(\sqrt{d_{i}}-\sqrt{1-d_{i}} \psi^{\dagger}\left(B_{i}\right) \psi_{h}^{\dagger}\left(A_{i}\right)\right)|A\rangle .
$$

Similar to a BCS state, where the entanglement structure of the modes pairs $A_{i}, B_{i}$ is clearly seen.

If we factor the single particle Hilbert space $H$ into

$$
H=\bigoplus_{i=1}^{N} \operatorname{span}\left\{A_{i}, B_{i}\right\} \bigoplus(\text { Complement }),
$$

the fermion Fock space factors into an appropriate tensor product. When working in terms of occupation number representation, the Fock space is spanned by states of the form $\left|\sigma_{A_{1}} \sigma_{B_{1}} \sigma_{A_{2}} \sigma_{B_{2}} \cdots\right\rangle \mid$ where $\sigma_{A_{i}}, \sigma_{B_{i}}=0$ or 1 is the occupation of state $A_{i}$ or $B_{i}$ respectively. In this basis we can write naturally the density matrix as

$$
\rho=|g s\rangle\left\langle g s\left|=\bigotimes_{i=1}^{N}\right| v_{i}\right\rangle\left\langle v_{i}\right|,
$$

where $v_{i}=\sqrt{d_{i}}\left|1_{A_{i}} 0_{B_{i}}\right\rangle+\sqrt{1-d_{i}}\left|0_{A_{i}} 1_{B_{i}}\right\rangle$. After a partial trace over the $B^{\prime} s$, the reduced density matrix is given by

$$
\rho_{A}=\bigotimes_{i=1}^{N}\left(\begin{array}{cc}
1-d_{i} & 0 \\
0 & d_{i}
\end{array}\right)
$$

where $d_{i}$ is the probability of having a particle in mode $A_{i}$.

From the density matrix $\rho_{A}$ we have

$$
\langle N\rangle_{A}=\operatorname{Tr}(M), \quad \Delta N_{A}^{2}=\left\langle N^{2}-\langle N\rangle^{2}\right\rangle_{A}=\operatorname{Tr} M(1-M) .
$$




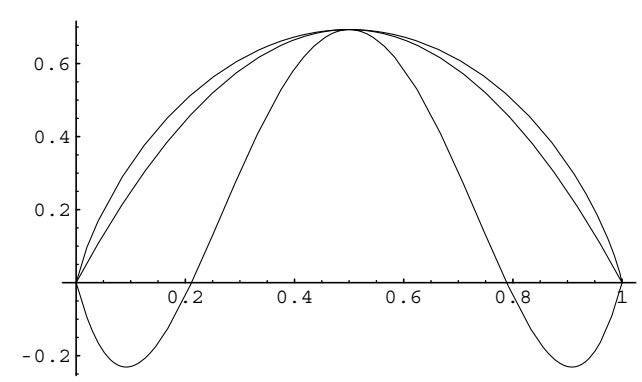

Figure 1. The functions $-x \log x-(1-x) \log (1-x) \geqslant 4 \log 2 x(1-x) \geqslant-8 \log 2 x(x-1)$ $\left(1-6 x+6 x^{2}\right)$ are related to the entropy, the 2 nd and 4 th cummulants.

To study further moments, denote $P(k)$ the probability of having $k$ fermions in $A$ and consider the generating function:

$$
\chi(\lambda)=\sum P(k) \mathrm{e}^{\mathrm{i} \lambda k}=\operatorname{det}\left(1+M\left(\mathrm{e}^{\mathrm{i} \lambda}-1\right)\right) .
$$

The cummulants of the number of fermions in $A$ may be extracted from the generating function by differentiating $\log \chi$ and setting $\lambda=0$. For example, the fourth cummulant is given by

$$
\left\langle\left\langle N^{4}\right\rangle\right\rangle=\left.\partial_{i \lambda}^{4} \log \chi\right|_{\lambda=0}=\operatorname{Tr}\left(M(1-M)\left(1-6 M+6 M^{2}\right)\right) .
$$

The reduced entropy is obtained from $\rho_{A}$ :

$$
S_{A}=-\operatorname{Tr}(M \log M+(1-M) \log (1-M)) .
$$

This entropy is equivalent to the usual entropy of a Fermi gas with the occupation number operator $M$.

Comparing the expressions (10), (12) and (13), and using the fact that $0 \leqslant M \leqslant 1$, we get the inequalities (1) as promised (figure 1).

The difference between the cummulants and the entropy comes from eigenstates with probability away from $1 / 2$ but not exactly 0 or 1 . The fourth cummulant may be used to estimate the contribution to the second moments of eigenvalues of $n_{A}$ away from $1 / 2$, as the function $-2 x(x-1)\left(1-6 x+6 x^{2}\right)$ is negative outside the interval $(1 / 2-\sqrt{3 / 4}, 1 / 2+\sqrt{3 / 4})$.

The inequalities are valid for finite temperatures too. To see this, consider a general density matrix of the form $\rho=Z^{-1} \mathrm{e}^{-K_{i j} a_{i}^{\dagger} a_{j}}$. By tracing out the $A^{\perp}$ degrees of freedom, the reduced density matrix acquires the form

$$
\rho_{A}=\operatorname{det}\left(1-n_{A}\right) \exp \left(\log \left(\frac{n_{A}}{1-n_{A}}\right)_{i j} a_{i}^{\dagger} a_{j}\right),
$$

where $n_{A}=P_{A} \frac{1}{1+\mathrm{e}^{K}} P_{A}$ is as an operator on $A$. The construction of this distribution from the covariance matrix $\left\langle a_{i} a_{j}^{\dagger}\right\rangle$ was discussed in several papers, mainly in the context of density matrix renormalization group [14, 15], where a similar expression was obtained.

The resulting density matrix on $A$ may be considered as thermal by appropriately choosing the 'energies' $\epsilon_{i}=\log \frac{1-d_{i}}{d_{i}}$. The density matrix may then be written as a 'thermal' state $\rho=Z_{A}^{-1} \exp \left(-\sum \epsilon_{i} \psi^{\dagger}\left(A_{i}\right) \psi\left(A_{i}\right)\right)$. Note that while the new eigenstates may have large values for the energy with respect to $K$, the operator $n_{A}$ is well defined, and bounded by 1 . The entropy is

$$
S=-\operatorname{Tr}\left(n_{A} \log n_{A}+\left(1-n_{A}\right) \log \left(1-n_{A}\right)\right) .
$$

The generating function $\chi(\lambda)$ becomes

$$
\chi(\lambda)=\operatorname{det}\left(1+n_{A}\left(\mathrm{e}^{\mathrm{i} \lambda}-1\right)\right)
$$

and the expressions for the moments are the same with the identification: $M \rightarrow n_{A}$. 
One may attempt to find a similar inequality for Bosons. For a closed system in thermal equilibrium

$$
S=\operatorname{Tr}\left(\left(1+n_{B E}\right) \log \left(1+n_{B E}\right)-n_{B E} \log n_{B E}\right)
$$

(where $n_{B E}$ is a Bose-Einstein occupation operator) and

$$
(\Delta N)^{2}=\operatorname{Tr} n_{B E}\left(1+n_{B E}\right)
$$

In most cases particle fluctuations will dominate the entropy, however one can check that whenever $n_{B E}<1$ (a dilute boson gas) then

$$
S_{\text {Bosons }} \geqslant(\log 2)(\Delta N)_{\text {Bosons }}^{2} .
$$

For systems in which the single particle energies have a gap $\Delta$ above the zero energy, as the temperature goes to zero we have $n_{i} \rightarrow 0$, making the inequality above valid.

The expressions above may be extended to systems with infinitely many particles. At zero temperature the Fermi sea defines a projection $P_{E}$, such as projection on momentum states below $k_{F}$, or the lowest Landau level in the example below. One may then replace $M$ by the operator $P_{E} P_{A} P_{E}$ in all expressions such as (10), (12) and (13). Note that whenever the average number of particles in the box $A$ is finite, the operator $P_{E} P_{A} P_{E}$ has a finite trace, i.e. it is a compact operator and as such may be regarded as a limit of finite matrices.

While the cases of spin chains and free fields were studied in numerous works, other important examples wait addressing. Here we consider the problem of $2 \mathrm{D}$ electrons in a quantum Hall sample.

The filled lowest Landau level is spanned by the states $|k\rangle=\frac{1}{\sqrt{\pi k !}} z^{k} \mathrm{e}^{-|z|^{2} / 2.4}$.

In [11], the entanglement entropy of a Chern-Simons theory describing a quantum hall defined on a disc was shown to scale like the radius. Let us now calculate the particle fluctuations. We choose $A$ to be a disc of radius $R$. Due to the radial symmetry of the system and of $A$, the factorization (5) is possible mode by mode, and the lowest Landau level may be written as

$$
|L L L\rangle=\prod_{k}\left(\sqrt{d_{k}} \psi^{\dagger}\left(|k\rangle_{A}\right)+\sqrt{1-d_{k}} \psi\left(|k\rangle_{A_{\perp}}\right)\right)|0\rangle,
$$

where $d_{k}=1-\frac{\Gamma\left(1+k, R^{2}\right)}{k !}$ are given in terms of the incomplete gamma function, $|k\rangle_{A}=$ $\frac{1}{\sqrt{d_{k}}} \chi(|z|<R)|k\rangle$, and $|k\rangle_{A_{\perp}}$ is defined similarly ${ }^{5}$. We will see that in this case both entropy and particle fluctuations are asymptotically linear in $R$, thereby proportional to the boundary area.

Particle fluctuations in this model are given by $=\left\langle\Delta N_{A}^{2}\right\rangle=\sum_{l} M_{l l}\left(1-M_{l l}\right)$. This sum may be approximated analytically as follows: write

$$
\sum_{l} M_{k k}^{2}=\sum_{k, l} \delta_{k, l} M_{k, l}=\frac{2}{\pi} \int_{0}^{2 \pi} \mathrm{d} \theta \int_{0}^{R} \int_{0}^{R} \mathrm{~d} x \mathrm{~d} y \mathrm{e}^{x^{2} \mathrm{e}^{-i \theta}+y^{2} \mathrm{e}^{i \theta}} x y \mathrm{e}^{-x^{2}-y^{2}} .
$$

This sum can be written, after the $x, y$ integrations, as the contour integral

$$
\sum_{l} M_{k k}^{2}=-\frac{1}{2 \pi \mathrm{i}} \oint_{|z|=1} \mathrm{~d} z \frac{\left(1-\mathrm{e}^{-R^{2}(1-z)}\right)\left(1-\mathrm{e}^{-R^{2}(1-1 / z)}\right)}{(1-z)^{2}} .
$$

${ }^{4}$ In the symmetric gauge for the vector potential $A_{i}=-\frac{B}{2} \epsilon_{i j} x^{j}$. The length unit is $\sqrt{2} l$ where $l=\sqrt{\frac{\hbar c}{e B}}$ is the magnetic length.

5 Note that the average number of particles contained in $A$ is $\sum d_{k} \sim R^{2}$. 


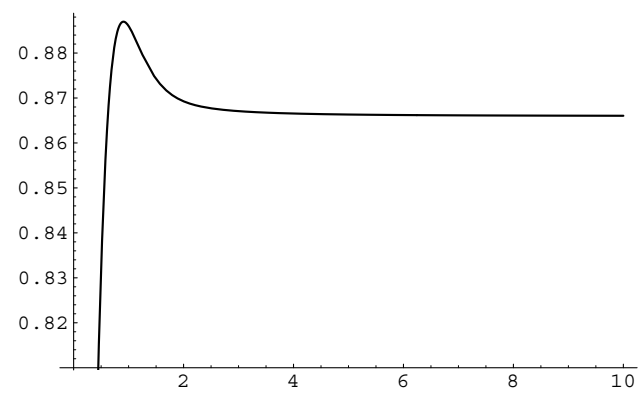

Figure 2. $S(R) / \Delta N(R)^{2}$ for a disc in the lowest landau level.

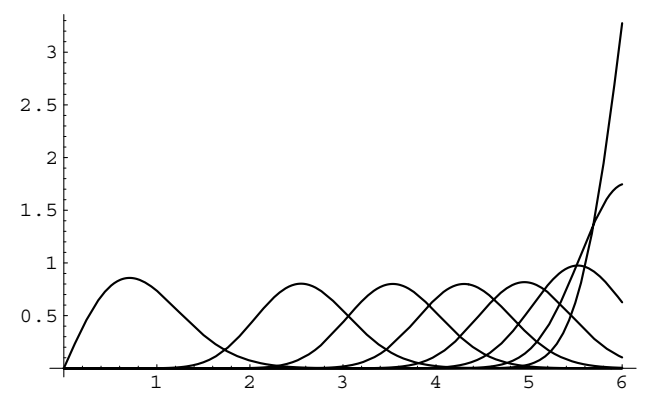

Figure 3. The modes $k=6 n, n=0, \ldots, 7$ for $R=6$ are concentrated closer to the boundary with increasing $k$.

Since the integrand is analytic outside $z=0$ we break it up and do part of the integration on a contour with $|z| \rightarrow \infty$ :

$(\Delta N(R))^{2}=R^{2}-\frac{1}{2 \pi \mathrm{i}} \oint_{|z| \rightarrow \infty} \mathrm{d} z \frac{\mathrm{e}^{-R^{2}}\left(\mathrm{e}^{R^{2} z}-\mathrm{e}^{R^{2} 1 / z}\right)}{(1-z)^{2}}-\frac{1}{2 \pi \mathrm{i}} \oint_{|z|=1} \mathrm{~d} z \frac{1-\mathrm{e}^{-R^{2}(2-z-1 / z)}}{(1-z)^{2}}$.

The first integral can be evaluated in the limit $R \rightarrow \infty$, by the residue theorem, and simply cancels the $R^{2}$ term. The contribution to the remaining integral comes from around the point $\theta=0$, we linearize near this point and get, as $R \rightarrow \infty$ :

$$
(\Delta N(R))^{2} \sim \frac{1}{2 \pi} \int_{-\infty}^{\infty} \mathrm{d} \theta \frac{1-\mathrm{e}^{-R^{2} \theta^{2}}}{\theta^{2}}=\frac{1}{\sqrt{\pi}} R .
$$

We see that in this model, the fluctuations are proportional to the boundary. A numerical check shows that the entropy and $\left\langle\Delta N_{A}^{2}\right\rangle$ have the same scaling (figure 2).

In this example it is manifest that for a fixed $R$, as $k$ grows larger (especially 6 for $k>R^{2}$ ), the modes $|k\rangle_{A}$ are localized stronger at the boundary, showing that the information is concentrated near the boundary (figure 3 ). Note that in this case we consider from the outset an infinite number of fermion modes, in contrast with the spin-chain case, where a finite segment yields a finite-dimensional fermionic Fock space after the Jordan-Wigner transformation.

We remark that since quasi-free fermionic states are related to determinantal processes [12], equivalent inequalities may be obtained for the connection of the entropy and the fluctuations in such systems. In particular, if one is interested in particle fluctuations, then

6 In this problem there is a crossover near $k=R^{2}$, where $d_{k} \sim 1 / 2$. To see this use the property $\frac{\Gamma(n, n)}{\Gamma(n)}<1 / 2<\frac{\Gamma(n, n-1)}{\Gamma(n)}$, from which $d_{R^{2}}\left(R^{2}\right)<1 / 2<d_{R^{2}}\left(R^{2}-1\right)$ (valid for $R^{2}$ integer). 
the theorem of Lebowitz and Costin [13] ensures a Gaussian behaviour for the scaled particle number fluctuations as the volume grows larger for a large class of determinantal processes.

In the case of a 1D Fermi gas, both entropy and particle fluctuations in a box of size $L$ scale as $\log k_{F} L^{7}$ particle number fluctuations in 1D conductors may be observed using ultra-fast transistors; however it will not be possible to ignore the Coulomb interaction. We note that for a $1 \mathrm{D}$ conductor, there is a pre-factor due to the cross section, which means that the fluctuations may be quite large. For the more general case of $d$ dimensions, we remark that the connection between fluctuations and entropy reported here was recently used in [16] to show that the entanglement entropy of free fermions scales faster than area law, i.e., as $L^{d-1} \log L$ for a hypercube with side $L$.

To summarize, in this letter we have shown how the ground state of fermions can be factored into sets of pairs of modes-inside and outside a given subsystem. We have outlined the connection between the entanglement entropy and the fluctuations in the particle number in two ways: first, the inequality (1) supplies a lower bound on the available entanglement entropy, and second, noting that in some cases (namely the 1D Fermi sea and the lowest Landau level) both quantities scale in a similar way.

\section{Acknowledgments}

I thank J D Bekenstein, J Lebowitz, L S Levitov, B Shapiro, A Retzker and M Reznikov for useful remarks.

\section{References}

[1] Bombelli L, Koul R K, Lee J and Sorkin R D 1986 Phys. Rev. D 34373

[2] Callan C G and Wilczek F 1994 Phys. Lett. B 33355

[3] Vidal G, Latorre J I, Rico E and Kitaev A 2003 Phys. Rev. Lett. 90227902

[4] Botero A and Reznik B 2004 Phys. Lett. A 33139

[5] Bekenstein J D 1981 Phys. Rev. D 23287

[6] DeMarco B and Jin D S 1999 Science 2851703

[7] Martin Th and Landauer R 1992 Phys. Rev. B 451742

[8] Levitov L S, Lee H W and Lesovik G B 1996 J. Math. Phys. 374845

[9] Avron J E, Elgart A, Graf G M and Sadun L 2002 J. Math. Phys. 43 3415-24

[10] Klich I and Levitov L at press

[11] Balachandran A P, Chandar L and Momen A 1997 Int. J. Mod. Phys. A 12 625-42

[12] Soshnikov A B 2000 J. Stat. Phys. 100491 Lyons R 2003 Pub. Mathématiques. de L'IHS 98 167-212

[13] Costin O and Lebowitz J L 1995 Phys. Rev. Lett. 75 69-72

[14] Peschel I 2003 J. Phys. A: Math. Gen. 36 L205 Chung M C and Peschel I 2001 Phys. Rev. B 64064412

[15] Cheong S and Henley C L 2003 Preprint cond-mat/0307172

[16] Gioev D and Klich I 2005 Preprint quant-ph/0504151

\footnotetext{
7 When disorder is present we expect the particle fluctuations to behave like $S=\log l_{e} k_{F}$, where $l_{e}$ is the elastic mean-free path.
} 